\title{
A!
}

This is an electronic reprint of the original article.

This reprint may differ from the original in pagination and typographic detail.

Vehkalahti, Roope; Pllaha, Tefjol; Tirkkonen, Olav

\section{Towards Ultra-Reliable Signature Coding With Multiple Transmit Antennas}

Published in:

Proceedings of IEEE 93rd Vehicular Technology Conference, VTC 2021

DOI:

10.1109/VTC2021-Spring51267.2021.9448780

Published: 15/06/2021

Document Version

Peer reviewed version

Please cite the original version:

Vehkalahti, R., Pllaha, T., \& Tirkkonen, O. (2021). Towards Ultra-Reliable Signature Coding With Multiple

Transmit Antennas. In Proceedings of IEEE 93rd Vehicular Technology Conference, VTC 2021 [9448780] (IEEE

Vehicular Technology Conference). IEEE. https://doi.org/10.1109/VTC2021-Spring51267.2021.9448780

This material is protected by copyright and other intellectual property rights, and duplication or sale of all or part of any of the repository collections is not permitted, except that material may be duplicated by you for your research use or educational purposes in electronic or print form. You must obtain permission for any other use. Electronic or print copies may not be offered, whether for sale or otherwise to anyone who is not an authorised user. 


\title{
Towards Ultra-Reliable Signature Coding With Multiple Transmit Antennas
}

\author{
Roope Vehkalahti, Tefjol Pllaha, Olav Tirkkonen \\ Department of Communication and Networking, Aalto University, Finland \\ Emails: \{roope.vehkalahti, tefjol.pllaha, olav.tirkkonen\} @ aalto.fi
}

\begin{abstract}
We consider sparse user detection in fading channels. With Rayleigh flat fading, deep fades occur with relatively high probability and it becomes challenging to provide highly reliable user detection, irrespective of the chosen multiuser detection algorithm. It has been proven that with a large number of receive antennas, this problem can be overcome and both the reliability and number of detectable users can be increased. In this paper, we show that similar improvements can be achieved by moderately increasing the number of transmit antennas at the user terminals. With multiple transmit antennas, code design becomes a problem. We provide a design criterion and show that the detection probability can be considerably improved by using the resulting well-balanced MIMO signature codes, especially in the high-reliability regime.
\end{abstract}

\section{INTRODUCTION}

The motivation for this work is to reach Ultra-Reliable Low Latency Communication (URLLC) [1] in a network with contention based access. Reaching URLLC is challenging, and it is well-known that in networks with scheduled resources, receive and transmit diversity methods are valuable for increasing reliability against uncertainties of fading channels [1]. Achieving URLLC in contention based access [2], [3] is an even more difficult task, however, due to the uncertainty caused by random user activity. Packet-level coding methods against random collisions have been considered in [3], and the frequency domain diversity arising from packet repetition has been analyzed in [4]. The role of physical layer transmit and receive diversity methods in contention based URLLC scenarios is not properly understood.

Here, we concentrate on the first step in contention based access; user detection in non-coherent fading channels, and consider physical layer code design. In the basic setup we have $N$ users of which a small number $K \ll N$ will randomly activate and try to indicate to a single receiver that they are active. One method for this is to use signature sequences, where each active user is sending a sequence of $n$ symbols. We assume that these signature sequences are pre-distributed to the users, and that each user is synchronized with the receiver. The number of signatures is larger than the number of orthogonal resources used, which leads to interference when multiple user are simultaneously active.

There exists an extensive literature on design of signature sequences, starting from early work on CDMA and culminating in a rich theory of signature code design principles both in synchronous and asynchronous scenarios [5], [6].

Recently, Compressed Sensing Multiuser Detection algorithms (CS-MUD) have been applied to the problem [7]-[12]. The theory of compressed sensing has both

This work was funded in part by the Academy of Finland (grants 299916 and 319484). suggested new signature code design principles and provided strong detection guarantees.

However, if the users channels are Rayleigh faded and unknown for the receiver, having highly reliable user detection is quite challenging, irrespective of the used detection method. Even in a scenario where there is only one active user there is a relatively high probability that the channel of the user is in a deep fade and the user is missed.

In such a scenario it becomes challenging to give good recovery guarantees. In the literature, limitations on the dynamic range of the fading coefficients [13, Thm. 2], [14, Thm 6.1], [15] are imposed to make guarantees possible, or the channel is assumed noiseless and a complex receiver structure is used [14, Thm. 4.4].

Limitations on the dynamic range and problems with receiver complexity can be avoided if the receiver has a large number of antennas and the physical channels between the receiver and the users are richly scattered. In [16] the authors analyzed coherence based deterministic codes and threshold detection. In this setting they found out that if the receiver has a large number of receive antennas it is enough to use length $n=O(K)$ codes for detecting $K$ users with high probability. For comparison, in a single antenna scenario, the best coherence based user detection guarantee for matching pursuit type algorithms requires $n=O\left(K^{2} \log \frac{N}{K}\right)$. This bound is referred as the "square-root bottleneck" [17].

More recently, in [18] and [19], it was proven that with a high number of receive antennas, a relatively low complexity decoder and random codes can be used to guarantee recovery with high probability when $n=O\left(\sqrt{K} \log \frac{N}{K}\right)$. This improves on the $O(K)$, which is the best known guarantee for any single antenna user detection method without noise [14, Thm. 4.4]. These works suggest that, while Rayleigh fading can be problematic in single antenna systems, it can be turned into a benefit when we have additional receive antennas in a rich scattering environment.

In this paper we investigate whether a similar effect can be achieved in the case where the users have additional transmit antennas, and if so, what kind of structure the signature codes should have. The motivation for the problem is clear. As seen earlier, large number of receive antennas is highly beneficial for user detection. However, there are use cases where massive antenna arrays can not be assumed, like vehicle-to-vehicle communication. As we will later see, if each of the users have $n_{t}$ transmit antennas the user detection capability of the receiver gets amplified so that it performs almost like having $n_{r} \times n_{t}$, instead of $n_{r}$ receive antennas. Hence additional transmit antennas allows us to operate closer to the massive regime without actual massive antenna arrays. 
If only one user is active at any given time the problem reduces to a non-coherent MIMO communication problem [20]. It is known that with proper code design one can improve the probability of correct user detection beyond single antenna signaturing. However, it is unclear if splitting the power between the transmit antennas and using MIMO signaturing is a good idea when more than one user can be active at the same time. This problem can be seen as a block sparse support recovery problem [21]. According to [21], a block sparse structure does help in signal recovery, but intentionally adding more variables to be recovered has a negative effect on overall recovery performance.

Here we consider a scenario where the fading coefficients have additional probabilistic structure. Furthermore, we are not interested in estimating channels; we only want to detect user activity. Following [16], we use the most simple threshold detector and a code design based on coherence [21]. Our results suggest that, with additional transmit antennas we can typically recover more active users. In section IV-B we show that already with two transmit antennas we can break the square-root bottle neck and get recovery guarantees with $n=O\left(K^{3 / 2} \ln N^{3 / 2+\varepsilon}\right)$. As the same proof works also in the case of additional receive antennas, we provide an extension to [16] by proving that already a small number of receive antennas allows us to partially overcome the square-root bottle neck.

Our probabilistic analysis is performed for a specific class of codes, which have been tailored for MIMO signaturing. The structure of these codes allows us to prove probability bounds and recovery guarantees. The codes are designed to minimize the block coherence following [21]. However, in order to fully benefit of the assumption that the fading coefficients are Gaussian, we also introduce an additional condition that the codebooks should be well-balanced in a specific manner.

Our results suggest that constructing well-balanced MIMO signature codebooks, with good coherence might be a new and interesting coding theoretic problem (see Section IV-A). Comparing well-balanced to non-balanced MIMO signature codes, simulations indicate an order of magnitude lower detection errors in the URLLC domain.

\section{SYSTEM MODEL}

Throughout the paper we are considering a scenario, where we have a set of users $\mathcal{U}$ indexed by $i$, each having $n_{t}$ transmit antennas. We will call a set of matrices $\mathcal{C} \subset \mathcal{M}_{n_{t} \times n}(\mathbb{C})$ a signature code designed for $\mathcal{U}$ if each element $X_{i} \in \mathcal{C}$ satisfies $\left\|X_{i}\right\|_{F}^{2}=1$, and if $|\mathcal{C}|=|\mathcal{U}|=N$. Transmission from user $i$ is of form $\sqrt{n \gamma} X_{i}$, where $\gamma$ is a constant that will be used to vary the transmission power. We assume that communication happens in a perfectly synchronized manner and in blocks of $n$ time units. We also assume that $\gamma$ is equal for all the users.

During a time-frame of $n$ units a random set $\mathcal{A} \subset \mathcal{U}$ of users is indicating activity by transmitting their code vector. The base station will then receive

$$
Y_{\mathcal{A}}=\sum_{i \in \mathcal{A}} \sqrt{n \gamma} H_{i} X_{i}+W
$$

where $H_{i}$ are independent $n_{r} \times n_{t}$ random matrices with i.i.d $\mathcal{C N}(0,1)$ coefficients and $W$ is an $n_{r} \times n$ random matrix with i.i.d $\mathcal{C} \mathcal{N}(0,1)$ coefficients. Here $n_{r}$ and $n_{t}$ are the number of receive and transmit antennas, respectively. In this signal model, $\gamma$ acts as the single-user Signal-to-Noise Ratio (SNR).
At any given time there are at most $K \ll N$ active users, and under this condition the receiver tries to identify these users. The receiver succeeds if it manages to recover exactly the set of all active users $\mathcal{A}$.

This problem can also be presented in the context of compressed sensing. For $n_{r}=1$, (1) can be written in an equivalent form as

$$
\mathbf{Y}_{\mathcal{A}}=\sqrt{n \gamma} \mathbf{X}_{\mathcal{C}} \mathbf{H}+\mathbf{W}
$$

where $\mathbf{X}_{\mathcal{C}}$ is an $n \times N n_{t}$ matrix, which consists of concatenation of matrices $X_{i}^{T}$ so that the first $n_{t}$ columns corresponds to the signature of the first user and so on. Here $\mathbf{H}$ is a sparse length $N n_{t}$ vector where the non-zero elements are i.i.d $\mathcal{C N}(0,1)$. With $n_{t}>1$ we have block sparsity; $\mathbf{H}$ is divided into consecutive blocks of $n_{t}$ elements, and inside each block either all the elements have non-zero value or all are zero.

If there are $|\mathcal{A}| \leq K$ active users, in the model (2) there are non-zero elements in at most $K$ blocks, and overall at most $n_{t} K$ non-zero elements. This is the block sparse model investigated in [21]. The problem of detecting which users are active is equivalent to finding which blocks have non-zero coefficients. This is the support recovery problem in the block sparse model, but with the additional assumption that $\mathbf{H}$ is not only block sparse, but also that the coefficients are Gaussian. If $n_{r}>1$ and $n_{t}=1$ the channel model (1) can be extended to an instance of the multi-channel compressed sensing problem [14]. Hence in full generality our work can be seen as a multi-channel block-sparse support recovery problem under additional data about the fading distribution of the coefficients.

We note that the recovery guarantees we will give later are for at most $K$ and not for exactly $K$ active users. Furthermore, while our bounds are probabilistic, they work for all sets with at most $K$ users and are not averages.

\section{SUPPORT RECOVERY OF WELL-BALANCED MIMO CODES}

In this section we introduce the concept of well-balanced MIMO signature codes and show how the small block coherence of such codes allows us to get strong recovery guarantees when using simple threshold decoder.

\section{A. Well-balanced MIMO Signature Codes and Their Coherence}

In [21] block sparse recovery was considered for signal detection, where the support (the active users) as well as the fading coefficients are estimated. However, as support recovery is an essential part of signal recovery, [21] can also be used as a guideline for finding good methods for our task.

We will denote the spectral norm of $A$ with $\rho(A)=$ $\sqrt{\lambda_{\max }\left(A A^{\dagger}\right)}$. Here $\lambda_{\max }\left(A A^{\dagger}\right)$ is the largest eigenvalue of the positive-semidefinite matrix $A A^{\dagger}$. Given a signature code $\mathcal{C} \subset M_{n_{t} \times n}(\mathbb{C})$ the concept of block-coherence of $\mathcal{C}[21]$ is defined as $\mu_{\mathcal{C}}=\max _{i \neq j} \rho\left(X_{i} X_{j}^{\dagger}\right)$.

In [21] it was proven that minimizing the block coherence does lead to good recovery guarantees. In our case we have the extra assumption that the coefficients are Gaussian random variables. As expected, we will see that minimizing the block coherence is a good idea also in this case. However, exploiting information about channel statistics leads to the additional condition that codes should be well-balanced. 
Definition 1. A MIMO signature code $\mathcal{C} \subset M_{n_{t} \times n}(\mathbb{C})$ is called well-balanced if for every $X_{i} \in \mathcal{C}$ we have

$$
X_{i} X_{j}^{\dagger}=c_{i, j} U_{i, j}
$$

where $U_{i, j}$ is an $n_{t} \times n_{t}$ unitary matrix and $c_{i, j}$ is a scalar. The signature code will be called $\varepsilon$-well-balanced if $\left|c_{i, i}\right|=1 / n_{t}$ and $\left|c_{i, j}\right| \leq \varepsilon$ for $i \neq j$.

Since all the eigenvalues of $U_{i, j}$ have absolute value 1 , for an $\varepsilon$-well-balanced MIMO signature code $\mathcal{C}$, we have that $\mu_{C}=$ $\max _{i \neq j}\left|c_{i, j}\right| \leq \varepsilon$. Thus we will refer to $\varepsilon$ as the coherence of the well-balanced code.

\section{B. Error probability of support recovery for well-balanced} codes

Let us now suppose we have a set of users $\mathcal{U}$ and an $\varepsilon$-well-balanced MIMO signature code $\mathcal{C} \subset \mathcal{M}_{n_{t} \times n}(\mathbb{C})$. We also suppose that the receiver uses a simple threshold decoder with threshold $s$. The threshold decoder for a set of active users $\mathcal{A}$ and channel model (1) works as follows. If $\left\|Y_{\mathcal{A}} X_{i}^{\dagger}\right\|^{2}>s$ the receiver decides that user $i$ is active, otherwise user $i$ is nonactive. Our result will be stated in a scenario, where at any given time there are at most $K$ active users. We will denote with $P_{\mathrm{e}}$ the probability that the receiver fails to recover the support of the transmission correctly. This probability is given in a form that holds for any subset $\mathcal{A}$ of $\mathcal{U}$ of size $|\mathcal{A}| \leq K$.

Before providing a bound for $P_{\mathrm{e}}$ in Theorem III we have to understand the probabilistic structure of $\left\|Y_{\mathcal{A}} X_{i}^{\dagger}\right\|^{2}$. Due to the space restrictions we will skip the proofs of Proposition 1 and Lemma 1.

Proposition 1. Consider the projection of the received signal (1) to a codeword,

$$
\left\|Y_{\mathcal{A}} X_{i}^{\dagger}\right\|_{F}^{2}=g Z \text {. }
$$

For any well-balanced code $\mathcal{C}, Z$ is a chi-squared random variable with $2 n_{t} n_{r}$-degrees of freedom and $g$ is a positive constant. If $i \notin \mathcal{A}$, then $g \leq \frac{\frac{1}{n_{t}}+n K \varepsilon^{2} \gamma}{2}$, and $g \geq \frac{\frac{1}{n_{t}}+n \gamma / n_{t}^{2}}{2}$ if otherwise.

Proposition 1 will be the key for providing error probability bounds for support recovery of well-balanced signature codes.

In what follows, we will denote with $p(i$,false $)$ the probability that given any set $\mathcal{A}$ of active users with $|\mathcal{A}| \leq K$ and a threshold $s$, the threshold decoder detects user $i$ as active although it was not. On the other hand we will denote with $p(i$, miss $)$ the probability that the threshold decoder, with threshold $s$ fails to detect that the user $i$ is active. One should note that the found bounds work for any user $i$ and set $\mathcal{A}$.

Lemma 1. If $i \notin \mathcal{A}$ and $\frac{s}{n_{r}\left(n n_{t} \varepsilon^{2} K \gamma+1\right)}>1$ then

$$
p(i, \text { false }) \leq\left(\frac{s e^{1-\frac{s}{n_{r}\left(n_{t} n \varepsilon^{2} K \gamma+1\right)}}}{n_{r}\left(n_{t} n \varepsilon^{2} K \gamma+1\right)}\right)^{n_{t} n_{r}} .
$$

$$
\begin{aligned}
& \text { If } i \in \mathcal{A} \text { and } \frac{s}{n_{r}\left(n \gamma / n_{t}+1\right)}<1 \text { then } \\
& \qquad p(i, \text { miss }) \leq\left(\frac{s e^{1-\frac{s}{n_{r}\left(n \gamma / n_{t}+1\right)}}}{n_{r}\left(n \gamma / n_{t}+1\right)}\right)^{n_{t} n_{r}} .
\end{aligned}
$$

Now we are ready to provide an upper bound for the probability that the threshold decoder with threshold $s$ fails to detect the correct set of active user $\mathcal{A}$. This bound holds for any set of active users $\mathcal{A}$ as long as $|\mathcal{A}| \leq K$.
Theorem 1. Consider an $\varepsilon$-well-balanced signature code $\mathcal{C}$ of Definition 1, a threshold detector with threshold s, maximum number $K$ of active users and code size $N$. If $\frac{s}{n_{r}\left(n \gamma / n_{t}+1\right)}<1$ and $\frac{s}{n_{r}\left(n_{t} n \varepsilon^{2} K \gamma+1\right)}>1$. Then the probability of erroneous recovery of the set of active users is

$P_{\mathrm{e}}<K\left(\frac{s e^{\left(1-\frac{s}{n_{r}\left(n \gamma / n_{t}+1\right)}\right)}}{n_{r}\left(n \gamma / n_{t}+1\right)}\right)^{n_{t} n_{r}}+N\left(\frac{s e^{1-\frac{s}{n_{r}\left(n_{t} n \varepsilon^{2} K \gamma+1\right)}}}{n_{r}\left(n_{t} n \varepsilon^{2} K \gamma+1\right)}\right)^{n_{t} n_{r}}$.

Proof. We have the set of active users $\mathcal{A},|\mathcal{A}| \leq K$. The probability that the threshold decoder fails in detecting this set of active users can be upper bounded as

$$
P_{\mathrm{e}} \leq \sum_{i \in \mathcal{A}} p(i, \text { miss })+\sum_{j \notin \mathcal{A}} p(j, \text { false }) .
$$

Using Proposition 1 we have upper bounds for the terms $p(i$, miss $)$ and $p(j$,false $)$ that are independent of $i$ and $j$. Further we know that there are at most $K$ terms in the first sum and at most $N$ in the second.

The error probability expression in Theorem 1 can be simplified to

$P_{\mathrm{e}}<K\left(\frac{s e}{n_{r}\left(n \gamma / n_{t}+1\right)}\right)^{n_{t} n_{r}}+N\left(\frac{s e^{1-\frac{s}{n_{r}\left(n_{t} n \varepsilon^{2} K \gamma+1\right)}}}{n_{r}\left(K n n_{t} \varepsilon^{2} \gamma+1\right)}\right)^{n_{t} n_{r}}$

Let us now set $s=f n_{r}\left(n_{t} n K \varepsilon^{2} \gamma+1\right)$, where $f>1$ is a freely chosen constant. With this choice of $s$ we automatically satisfy the second condition in Theorem 1. The first condition holds if

$$
\frac{f n_{r}\left(n n_{t} K \varepsilon^{2} \gamma+1\right)}{n_{r}\left(n \gamma / n_{t}+1\right)}<1
$$

and we have the following corollary.

Corollary 1. If there are at most $K$ active users, the probability of error for recovering the correct support is upper bounded by

$$
P_{\mathrm{e}}<K\left(\frac{e f\left(K n_{t} n \varepsilon^{2} \gamma+1\right)}{\left(n \gamma / n_{t}+1\right)}\right)^{n_{t} n_{r}}+N\left(f e^{1-f}\right)^{n_{t} n_{r}} .
$$

where $f>1$ is a freely chosen parameter.

We note that condition (6) does not limit the use of our bound at all as in order to make the first term of the right side in (7) smaller than one we have to satisfy condition (6).

For large values of $\gamma$ Equation (7) can be approximated as

$$
P_{\mathrm{e}}<K\left(f K e n_{t}^{2} \varepsilon^{2}\right)^{n_{t} n_{r}}+N\left(f e^{1-f}\right)^{n_{t} n_{r}} .
$$

This reveals how minimizing $\varepsilon$ leads to strong recovery guarantees. If on the right side of the equation we select $f$ large enough to push the rightmost term towards zero, then $\varepsilon$ has to be small enough so that the term on left will be small as well.

Remark 1. If we analyze the error probability of a fixed code with coherence $\varepsilon$, Equation (8) reveals the effect of additional receive antennas. Assuming we have selected $f$ and $K$ in such a way that $\left(f K e n_{t}^{2} \varepsilon^{2}\right)^{n_{t} n_{r}}<1$ and $\left(f e^{1-f}\right)^{n_{t} n_{r}}<1$, then in (8) additional receive antennas will push the error probability towards zero exponentially.

Adding transmit antennas has a similar effect to the error probability. However, as $f K e n_{t}^{2} \varepsilon^{2}$ includes the term $n_{t}^{2}$, the effect of additional antennas should be compensated with smaller $\varepsilon^{2}$ or smaller $K$. Furthermore, changing the number of transmit antennas requires changing the code. Hence we 
can not guarantee that $N$ and $\varepsilon^{2}$ will remain the same when we increase the number of transmit antennas. In Section IV-B we will see that despite these issues using additional transmit antennas is beneficial and the effect is indeed similar to that of additional receive antennas.

\section{MIMO SIGNATURE CODES FROM MUBS}

In the previous section we found out how small coherence of an $\varepsilon$-well-balanced MIMO signature code provides good recovery guarantees. However, this analysis did not directly answer whether it is better to use one transmit antenna or more. In this section we consider two families of signature codes. One for one transmit antenna and the other for two, and show that with two transmit antennas we get better performance. The coherence of the used single antenna codes is close to the Welch bound. Asymptotically Welch bound is in class $\Theta\left(\frac{1}{\sqrt{n}}\right)$. Our analysis therefore reveals that even if a single antenna code reaches this bound it can not have as good recovery guarantee as our simple two antenna code does.

\section{A. Construction of well-balanced codes}

Unitary matrices $X_{i} \in \mathcal{M}_{n}(\mathbb{C})$ are called mutually unbiased bases (MUBs) if the matrix of absolute values of row inner products satisfies $\left|X_{i} X_{j}^{\dagger}\right|=\mathbb{1} / \sqrt{n}$, where $\mathbb{1}$ is the all-ones matrix. It is well-known that in power-of-two dimensions $n=$ $2^{k}$ there exist $n+1$ MUBs, and this number is maximal. In this paper we will work with the maximal collection of Kerdock MUBs [23]. We will denote $\mathcal{C}_{n}$ the set of $n(n+1)$ unit norm vectors that are formed by the rows of the Kerdock MUBs. Such code is trivially a $\varepsilon$-well-balanced signature code for the case $n_{r}=n_{t}=1$ with coherence $\varepsilon=1 / \sqrt{n}$.

We are most interested in a true MIMO scenario in which $n_{t}, n_{r}>1$. For the case $n_{t}=2$ we will construct a $\varepsilon$-well-balanced signature code $\mathcal{C}_{n, 2} \subset \mathcal{M}_{2 \times n}(\mathbb{C})$ as follows. For each $x_{i} \in \mathcal{C}_{n / 2}$, we will denote

$$
X_{i}:=\frac{1}{\sqrt{2}}\left(\begin{array}{cc}
x_{i} & 0 \\
0 & x_{i}
\end{array}\right) \in \mathcal{C}_{n, 2} .
$$

It is readily verified that $\mathcal{C}_{n, 2}$ is a $\varepsilon$-well-balanced MIMO signature code with coherence $\varepsilon=1 / \sqrt{2 n}$ and size $\left|\mathcal{C}_{n, 2}\right|=$ $n / 2(n / 2+1)$. In general, if $n_{t}$ is a power of two then $\mathcal{C}_{n, n_{t}} \subset \mathcal{M}_{n_{t}, n}$ can be constructed similarly using signatures $x_{i} \in \mathcal{C}_{n / n_{t}}$.

Remark 2. Constructing well-balanced codes for a single antenna is a conventional code design problem. In this case one is interested on codes with small coherence or large size. The problem is well-studied from the context of sequence design [24], and there exist several bounds. In the MIMO context the idea of using MUBs for constructing codes for single user non-coherent MIMO has appeared in [20], but the user detection problem was not considered. Using MIMO spreading sequences in coherent CDMA MIMO has appeared in [25] and as mentioned block coherence has been discussed in the context of compressed sensing in [21]. However none of these works considered the random access problem in quasi-static fading channels and as far as we know there is no previous work on well-balanced MIMO signature codes.

Remark 3. For $n=8$, we have $\left|\mathcal{C}_{8,2}\right|=20$ and coherence $\varepsilon=1 / 4$. The underlying set of single antenna signatures also achieves the orthoplex bound [26] proving that for coherence $1 / 4$ this is the largest possible set of diagonal well balanced codes. However, in this case we are aware of a well-balanced code with the same coherence, but of size 28. Thus, one can go beyond the diagonal constructions that we are using in this paper.

\section{B. Asymptotic performance analysis}

Let us now consider a scenario, where the receiver has a single antenna, but the transmitters have an option to select between using one or two antennas. To simplify the comparison, we assume that there is no noise. The signaturing methods we compare are from the previous section. For the single transmit antenna case we have a signature code $\mathcal{C}_{n}$ with $n(n+1)$ elements and coherence $\varepsilon_{1}=1 / \sqrt{n}$. For the two transmit antennas we use the code $\mathcal{C}_{n, 2}$ with $n / 2(n / 2+1)$ elements and coherence $\varepsilon_{2}=1 / \sqrt{2 n}$. In order to have a fair comparison, we select the same number $N$ of codewords from both codes. For a single transmit antenna, (8) reads as

$$
P_{\mathrm{e}}<K\left(\frac{f K e}{n}\right)+N\left(f e^{1-f}\right),
$$

whereas for the two transmit antenna case we have

$$
P_{\mathrm{e}}<K\left(\frac{2 f K e}{n}\right)^{2}+N\left(f e^{1-f}\right)^{2} .
$$

By analysing the conditions which $f$ must satisfy so that we can asymptotically guarantee support recovery of at most $K$ users, we find that for a single transmit antenna we must have at least $K=O\left(\frac{n^{1 / 2}}{\ln N^{1 / 2}}\right)$. With two transmit antennas we can set $f=\ln N$ and achieve correct support recovery already when $K=\Theta\left(\frac{n^{2 / 3}}{(\ln N)^{2 / 3+\varepsilon}}\right)$. It follows that we can guarantee support recovery when $n=O\left(K^{3 / 2} \ln N^{3 / 2+\varepsilon}\right)$.

We note that the single antenna code suffers from the square-root bottleneck [17] that limits the recovery capability of coherence based greedy methods. However, with two transmit or receive antennas we can break this limitation.

\section{Simulated performance}

In the previous section our asymptotic analysis suggested that using additional transmit antennas and well-balanced codes gives better recovery guarantees. In this section we are testing this by a simulation. We compare the performance of the well-balanced codes $\mathcal{C}_{512,2}$ and $\mathcal{C}_{512,4}$ with single transmit antenna code $\mathcal{C}_{512}$ for 2 and 4 receive antennas. For a reference we also measured the performance of $\mathcal{C}_{512}$ with a single receive antenna.

In order to demonstrate the benefits of codes being well-balanced we also included the performance of non-balanced 2-transmit antenna code $\mathcal{D}_{512,2}$ and a non-balanced 4-transmit antenna code $\mathcal{D}_{512,4}$ that were constructed as follows. Starting with MUBs in 512 dimensions, we select consecutive rows of such MUBs to create signatures $Y_{i} \in \mathcal{M}_{\ell \times 512}$. Then $\mathcal{D}_{512, \ell}$ consists of $\left|\mathcal{C}_{512, \ell}\right|$ such signatures.

Since the actual code sizes are massive, we have selected the size of the user population to be $N=5000$, so that we can study the performance of the codes in the ultra-reliability region. The SNR is set to $20 \mathrm{~dB}$.

For the simulations we assume to know the number of active users $K$, and exhaustively find the $K$ highest values of $\left\|Y_{\mathcal{A}} X_{i}^{\dagger}\right\|_{F}$. If $p_{k}$ denotes the probability to correctly recovering 
$k$ users, then the single transmission error probability is measured by

$$
p_{O}=1-\frac{1}{K} \sum_{i=1}^{k} k p_{k} .
$$

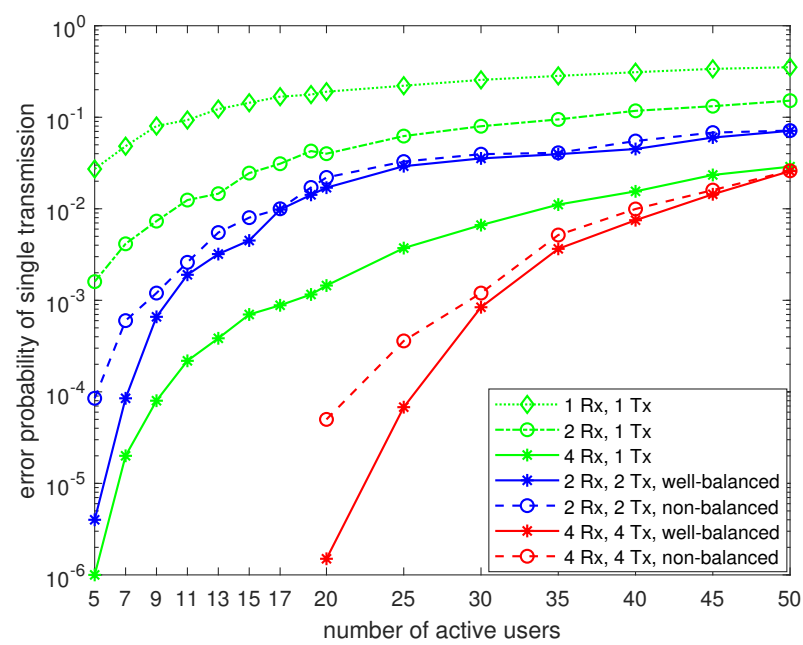

Fig. 1. Comparison of well-balanced codes with non-balanced codes.

From the simulations we can see that, as expected, using more transmit antennas does provide considerable gain. In all cases with the same diversity degree, we see that it is more beneficial to increase the number of receive antennas than transmit antennas, as is indicated by the bounds in Theorem 1 . Overall, we see a remarkable gain in the supported number of users, when transmit and receive diversity is used for signature coding. The gain from using a well-balanced multi-antenna signature is considerable. Especially in the high-reliability regime, a well-balanced code can provide an order of magnitude smaller error probability than a non-balanced code with similar block coherence.

\section{CONCLUSION}

In this paper, we introduced the notion of $\varepsilon$-well-balanced MIMO signature codes for user activity detection. For such codes, we could provide detection guarantees for larger sets of active users than is possible with corresponding single antenna techniques. The effect is especially pronounced in the high-reliability regime. We point out that our work demonstrates that multiple transmit antennas are beneficial in the specific case of coherence based codes and a threshold decoder. In future works, we will study code constructions and bounds of $\varepsilon$-well-balanced MIMO signature codes, and their performance under more advanced CS algorithms.

\section{REFERENCES}

[1] G. Durisi, T. Koch and P. Popovski, "Toward massive, ultrareliable, and low-latency wireless communication with short packets," Proc. IEEE, vol. 104, no. 9, pp. 1711-1726, Sept. 2016.

[2] B. Singh, O. Tirkkonen, Z. Li and M. Uusitalo, "Contention-based access for ultra-reliable low latency uplink transmissions," IEEE Wireless Commun. Letters, vol. 7, no. 2, pp. 182-185, Apr 2018.

[3] C. Boyd, R. Vehkalahti, and O. Tirkkonen, "Combinatorial code designs for ultra-reliable IoT random access," in Proc. IEEE Int. Symp. on Pers., Ind. and Mobile Radio Comm., Oct. 2017, pp. 1-5.

[4] C. Boyd, R. Kotaba, O. Tirkkonen and P. Popovski, "Non-orthogonal contention-based access for URLLC Devices with frequency diversity," Signal Proc. Advances for Wireless Commun., pp.1-5, July 2019.
[5] D. V. Sarwate and M. B. Pursley, "Cross-correlation properties of pseudo-noise and related sequences," Proc. IEEE, vol. 68, pp. 598-619, May 1980.

[6] M. Rupf, J. L. Massey, "Optimum sequence multisets for synchronous code-division multiple-access channels", IEEE Tran. Inform. Theory, Vol. 40, pp. 1261-1266, 1994.

[7] H. Zhu and G. B. Giannakis, "Exploiting sparse user activity in multiuser detection," IEEE Trans. Commun., vol. 59, no. 2, pp. 454-465, Feb. 2011.

[8] H. F. Schepker and A. Dekorsy, "Sparse multi-user detection for cdma transmission using greedy algorithms," 2011 8th International Symposium on Wireless Communication Systems, pp. 291-295, Nov. 2011.

[9] L. Applebaum, W. U. Bajwa, M. F. Duarte, and R. Calderbank, "Asynchronous code-division random access using convex optimization," Physical Communication, vol. 5, no. 2, pp. 129 - 147, 2012.

[10] Z. Utkovski, T. Eftimov, and P. Popovski, "Random access protocols with collision resolution in a noncoherent setting," IEEE Wireless Commun. Lett., vol. 4, pp. 445-448, Aug. 2015.

[11] Z. Chen, F. Sohrabi, and W. Yu, "Sparse activity detection for massive connectivity," IEEE Trans. Signal Process., vol. 66, no. 7, pp. 1890-1904, Apr. 2018

[12] Y. Ji, C. Stefanovic, C. Bockelmann, A. Dekorsy, and P. Popovski, "Characterization of coded random access with compressive sensing based multi-user detection," in IEEE GLOBECOM, pp. 1740-1745, Dec. 2014.

[13] K. Schnass and P. Vandergheynst, "Average performance analysis for thresholding", in IEEE Signal Process. Lett., vol. 14, no. 11, pp. 828-831, Nov. 2007.

[14] Y. C. Eldar and H. Rauhut, "Average case analysis of multichannel sparse recovery using convex relaxation", IEEE Trans. on Inform. Theory, vol. 56, no. 1, pp. 505-519, Jan. 2010.

[15] E. Miandji, M. Emadi, J. Unger and E. Afshari, "On probability of support recovery for orthogonal matching pursuit using mutual coherence", IEEE Signal Process. Lett., vol. 24, no. 11, pp. 1646-1650, Nov. 2017.

[16] R. Gribonval, H. Rauhut, K. Schnass, and P. Vandergheynst, "Atoms of all channels, unite! Average case analysis of multi-channel sparse recovery using greedy algorithms," J. Fourier Anal. Appl., vol. 14, no. 5, pp. 655-687, 2008

[17] Y. Chi and R. Calderbank, "Coherence-based performance guarantees of orthogonal matching pursuit", 50th Annual Allerton Conference on Communication, Control, and Computing (Allerton), Monticello, IL, 2012, pp. 2003-2009.

[18] L. Liu and W. Yu, "Massive connectivity with massive MIMO-part I: Device activity detection and channel estimation,'IEEE Trans. Signal Process., vol. 66, no. 11, pp. 2933-2946, June, 2018.

[19] S. Haghighatshoar, P. Jung, and G. Caire, "A new scaling law for activity detection in massive MIMO systems," ArXiv eprints, Mar. 2018.

[20] B. M. Hochwald and T. L. Marzetta, "Unitary space-time modulation for multiple-antenna communications in Rayleigh flat fading", IEEE Trans. on Inform. Theory, vol. 46, no. 2, pp. 543-564, March 2000.

[21] Y. C. Eldar, P. Kuppinger and H. Bölcskei, "Block-sparse signals: uncertainty relations and efficient recovery", in IEEE Trans. Signal Process., vol. 58, no. 6, pp. 3042-3054, June 2010.

[22] S. Dasgupta and A. Gupta, "An elementary proof of a theorem of Johnson and Lindenstrauss", Random Structures and Algorithms, vol. 22, pp. $60-65,2003$

[23] A. Calderbank, P. Cameron, W. Kantor, and J. Seidel, " $\mathbb{Z}_{4}$-Kerdock codes, orthogonal spreads, and extremal Euclidean line-sets", Proc. London Math. Soc., vol. 75, pp. 436-480, Sep 1997.

[24] J. H. Conway, R. H. Hardin and N. J. A. Sloane, "Packing lines, planes, etc.: Packings in Grassmannian spaces", Experimental Mathematics 5, pp. 139-159, 1996.

[25] B. Hochwald, T. L. Marzetta and C. B. Papadias, "A transmitter diversity scheme for wideband CDMA systems based on space-time spreading", IEEE J. Sel. Areas Commun., vol. 19, no. 1, pp. 48-60, Jan 2001.

[26] R. A. Rankin, "The closest packing of spherical caps in $n$ dimensions", Proc. Glasgow Math. Assoc., pp. 139-144, 1955. 